\title{
Reflexiones para medir el impacto socio económico dell bono productivo alimenticio
}

\section{Reflections to measure the socioeconomic impact of the food production bonus}

\author{
Mondragón-Laínez, Ana Doris; Zúniga-González, Carlos Alberto; \\ Editor Academico Prof. Dr. Juan Manuel Zaldívar Cruz
}

\author{
Ana Doris Mondragón-Laínez \\ anamonlay7@yahoo.es \\ Universidad Nacional Autónoma de Nicaragua, \\ Nicaragua \\ Carlos Alberto Zúniga-González \\ czuniga@ct.unanleon.edu.ni \\ Universidad Nacional Autónoma de Nicaragua, \\ UNAN-León. , Nicaragua \\ Editor Academico Prof. Dr. Juan Manuel Zaldívar \\ Cruz \\ Colegio Postgraduados Mexico, México
}

\section{Revista Iberoamericana de Bioeconomía y Cambio Climático \\ Universidad Nacional Autónoma de Nicaragua, León, Nicaragua ISSN-e: 2410-7980 \\ Periodicidad: Semestral \\ vol. 3, núm. 6, 2017 \\ czuniga@ev.unanleon.edu.ni}

Recepción: 06 Junio 2016

Aprobación: 02 Agosto 2016

URL: http://portal.amelica.org/ameli/
jatsRepo/394/3941753002/index.html

DOI: https://doi.org/10.5377/ribcc.v3i6.5950

Autor de correspondencia: anamonlay7@yahoo.es
Resumen: El presente estudio es una revisión del diseño metodológico para medir el impacto socio económico del programa del Bono Productivo. Así, cuando se estudia una metodología lo primero que se encuentra el investigador es la definición del tipo de investigación que desea realizar. La escogencia del tipo de investigación determinará los pasos a seguir del estudio, sus técnicas y métodos que puedan emplear en el mismo. En general determina todo el enfoque de la investigación influyendo en instrumentos, y hasta la manera de cómo se analiza los datos recaudados para medir un impacto como el del Bono Productivo. Así, el punto de los tipos de investigación en una investigación va a constituir un paso importante en la metodología, pues este va a determinar el enfoque del mismo. Este puede dividirse en dos tipos principales de Campo o de Laboratorio. Que a su vez puede clasificarse en cuatro tipos principales: Estudios Exploratorios: También conocido como estudio piloto, son aquellos que se investigan por primera vez o son estudios muy pocos investigados. También se emplean para identificar una problemática. Estudios: Describen los hechos como son observados. Estudios Correlaciónales: Estudian las relaciones entre variables dependientes independientes, ósea se estudia la correlación entre dos variables. Estudios Explicativos: Este tipo de estudio busca el por qué de los hechos, estableciendo relaciones de causa- efecto. Pero en este caso estaremos abordando todo los apartados que conllevan el diseño metodológico de la investigación, cualitativa y los aspectos que a ella se refieren.

Palabras clave: Diseño metodológico, Investigación cualitativa, tipo de investigación explicativa, Método, instrumentos, muestra.

Abstract: The present study is a review of the methodological design to measure the socio-economic impact of the Productive Bonus program. So when I know studies a methodology the first thing the researcher finds is the definition of the type of research you want to carry out. The choice of type research will determine the steps to be followed in the study, its techniques and methods they can use in it. In general it determines the whole research approach influencing instruments, and even the way of how the data collected is analyzed to measure an impact such as that of the Bond Productive. Thus, the point of investigation 
types in an investigation goes to constitute an important step in the methodology, as this will determine the focus of it. This can be divided into two main types of Field or from laboratory. Which in turn can be classified into four main types: Exploratory Studies: Also known as a pilot study, are those that are being investigated for the first time or are very few studies investigated. They are also used to identify a problem. Studies: Describe the facts as they are observed. Correlational Studies: Study the relationships between dependent variables independent, bone, the correlation between two variables is studied. Studies Explanatory: This type of study looks for the why of the facts, establishing cause-effect relationships. But in this case we will be addressing all the sections involved in the methodological design of the research, qualitative and the aspects that refer to it.

\section{INTRODUCCION}

Hernández, Fernández y Baptista (2003) establecen estos cuatro tipos de investigación, basándose en la estrategia de investigación que se emplea, ya que "el diseño, los datos que se recolectan, la manera de obtenerlos, el muestreo y otros componentes del proceso de investigación son distintos en estudios exploratorios, descriptivos, correlacionales y explicativos" (p. 114). No obstante, existen otras maneras de clasificar los tipos de investigaciones, por ejemplo se pueden clasificar según: El proceso formal. Este se refiere al método que se emplea en el estudio, se divide en:

Método deductivo: Parte de una premisa general para obtener las conclusiones de un caso particular. Pone el énfasis en la teoría, modelos teóricos, la explicación y abstracción, antes de recoger datos empíricos, hacer observaciones o emplear experimentos.

Método inductivo: Se analizan solo casos particulares, cuyos resultados son tomados para extraer conclusiones de carácter general. A partir de las observaciones sistemáticas de la realidad se descubre la generalización de un hecho y una teoría. Se emplea la observación y la experimentación para llegar a las generalidades de hechos que se repiten una y otra vez.

Método hipotético-deductivo: A través de observaciones realizadas de un caso particular se plantea un problema. Éste lleva a un proceso de inducción que remite el problema a una teoría para formular una hipótesis, que a través de un razonamiento deductivo intenta validar la hipótesis empíricamente.

Según el grado de abstracción: Este se divide en: Investigación pura (básica): Esta investigación busca aumenta la teoría, por lo tanto se relaciona con nuevos conocimientos, de este modo no se ocupa de las aplicaciones prácticas que puedan hacer referencias los análisis teóricos.

Investigación aplicada: Su principal objetivo se basa en resolver problemas prácticos, con un margen de generalización limitado. De este modo genera pocos aportes al conocimiento científico desde un punto de vista teórico (Hernández \& Fernández, 2003).

Según el grado de generalización: Se divide en:

Investigación fundamental: A partir de la muestra de sujetos, las conclusiones de la investigación se hace extensiva a la población y se orienta a las conclusiones. Su objetivo se centra en el aumento de información teórica y se relaciona con la investigación pura (básica).

\section{NotAS DE AUTOR}


Investigación acción: Se centra en generar cambios en una realidad estudiada y no coloca énfasis en lo teórico. Trata de unir la investigación con la práctica a través de la aplicación, y se orienta en la toma de decisiones y es de carácter ideográfico.

Según la naturaleza de los datos: Su división es:

Metodología cuantitativa: Para cualquier campo se aplica la investigación de las Ciencias Físico-Naturales. El objeto de estudio es "externo" al sujeto que lo investiga tratando de lograr la máxima objetividad. Intenta identificar leyes generales referidas a grupos de sujeto o hechos. Sus instrumentos suelen recoger datos cuantitativos los cuales también incluyen la medición sistemática, y se emplea el análisis estadístico como característica resaltante.

Metodología cualitativa: Es una investigación que se basa en el análisis subjetivo e individual, esto la hace una investigación interpretativa, referida a lo particular.segun la naturaleza de las variables en : Se centra en la manera como se desea controlar o no las variables. Se divide en: Investigación descriptiva: No hay manipulación de variables, estas se observan y se describen tal como se presentan en su ambiente natural. Su metodología es fundamentalmente descriptiva, aunque puede valerse de algunos elementos cuantitativos y cualitativos.

Investigación experimental: Se manipula una o varias variables independientes, ejerciendo el máximo control. Su metodología es generalmente cuantitativa.

Investigación "ex post facto": No se controlan las variables independientes, dado que el estudio se basa en analizar eventos ya ocurridos de manera natural. Como el evento ya ha ocurrido los métodos de análisis pueden ser descriptivos o experimentales. Y según la naturaleza de los objetivos; Se refiere en cuanto al nivel de conocimiento que se desea alcanzar. Esta se divide en: Investigación exploratoria: Es considerada como el primer acercamiento científico a un problema. Se utiliza cuando éste aún no ha sido abordado o no ha sido suficientemente estudiado y las condiciones existentes no son aún determinantes.

Investigación Descriptiva: Se efectúa cuando se desea describir, en todos sus componentes principales, una realidad.

Investigación correlacionar: Es aquel tipo de estudio que persigue medir el grado de relación existente entre dos o más conceptos o variables.

Investigación explicativa: Es aquella que tiene relación causal, no sólo persigue describir o acercarse a un problema, sino que intenta encontrar las causas del mismo. Puede valerse de diseños experimentales y no experimentales.

Investigación experimental: El objetivo se centra en controlar el fenómeno a estudiar, emplea el razonamiento hipotético-deductivo. Emplea muestras representativas, diseño experimental como estrategia de control y metodología cuantitativa para analizar los datos.

Investigación predicativa: Se plantea predecir fenómenos o hechos basándose en datos anteriores y técnicas cuantitativas tales como regresión múltiple o análisis causal.

Según Hernández, Fernández, \& Baptista (2006). La investigación Es un proceso creativo En el se encuentran dificultades imprevistas y de asechanzas paradójicas, de prejuicios invisibles y de obstáculos de todo tipo. Todos ellos conllevan a formar parte del apartado de mayor relevancia dentro del proceso de investigación.

\section{DESARROLLO}

La metodología es el instrumento que enlaza el sujeto con el objeto de la investigación, Sin la metodología es casi imposible llegar a la lógica que conduce al conocimiento científico Se puede definir como la descripción, el análisis y la valoración crítica de los métodos de investigación Dentro del método caben los procedimientos y técnicas más específicas que se emplean en las investigaciones, El método científico es un modelo general de 
acercamiento a la realidad, una especie de pauta o matriz que es muy abstracta y amplia (Hernandez Sampieri et al., 1991).

Se necesita una manera sistemática, controlada, empírica y crítica para llevar a cabo cualquier procedimiento. Porque la mayoría de las ideas iniciales de una investigación son normalmente vagas e imprecisas. Es necesario transformar los planteamientos iniciales en forma más precisa y estructurada.

Méndez, Ignacio, et al, (2001), afirma que Diseño del Protocolo de Investigación constituye la etapa de planificación de una investigación. También es el documento base del investigador, cuyas especificaciones le permiten orientar el proceso de ejecución del trabajo. Es el documento que contiene el plan de un proyecto de investigación científica, con el máximo posible de detalle, precisión y claridad Diseño metodológico:

El marco metodológico es una relación clara y concisa de cada una de las etapas de la investigación. En términos generales, el diseño metodológico es la descripción de cómo se va a realizar la investigación, el cual incluye:

- Tipo de investigación

La tipología se refiere al alcance que puede tener una investigación científica. La estrategia de investigación depende del tipo de estudio que se elija, ya que éste determina el diseño, los datos que se recolecten, la manera de obtenerlos, el muestreo y otros componentes del proceso de investigación.

- Formulación de la hipótesis y definición de las variables

Las hipótesis son proposiciones tentativas acerca de las relaciones entre dos o más variables y se apoyan en los conocimientos organizados y sistematizados. No necesariamente son verdaderas, pueden o no serlo, pueden o no comprobarse con hechos. Son explicaciones tentativas y no todos los estudios plantean hipótesis. Las variables por su parte son una propiedad que puede variar y cuya variación es susceptible de medirse, selección de la muestra

para seleccionar una muestra apropiada se deben tomar en cuenta los siguientes criterios:

Definir los sujetos u objetos de estudio delimitar la población, elegir el tipo de muestra. Definir el tamaño de la muestra, aplicar el procedimiento de selección Métodos y técnicas de investigación Para obtener información sobre un mismo problema pueden emplearse diferentes métodos y técnicas, sin embargo, lo relevante de la investigación científica radica en seleccionar los adecuados, dependiendo de la naturaleza del fenómeno, los objetivos del estudio y la perspectiva de análisis. Los métodos y técnicas son las herramientas metodológicas de la investigación. El método es la manera de alcanzar los objetivos o el procedimiento para ordenar la actividad. Cabe destacar que el método se desprende de la teoría. La técnica es un conjunto de reglas y operaciones para el manejo de los instrumentos que auxilia al individuo en la aplicación de los métodos. Análisis e interpretación de los datos El procedimiento del análisis de los datos incluye los siguientes pasos: Toma de decisiones respecto a los análisis por realizar (pruebas estadísticas)

Elaboración del plan de análisis Ejecución del plan Obtención de los resultados h. Referencias bibliográficas: Es un listado de las referencias bibliográficas citadas o consultadas para la elaboración del protocolo, escritas de acuerdo con la normativa internacional para citación de bibliografía. y su Cronograma:

Describe las actividades por realizar en el tiempo. La distribución en el tiempo depende de la programación de las actividades, puede ser en semanas, meses o años. Recursos:

Especifica la fuente, el rubro de presupuesto y los recursos necesarios para llevar a cabo el proyecto

Los protocolos de ensayos clínicos requieren de información específica, fundamentalmente relacionada con el producto en estudio y la protección de los individuos que son sujetos de experimentación, tal como se indica en el siguiente apartado.

Mientras que Paz, M. (2003). la investigación cualitativa atraviesa diversas disciplinas, participa de una gran variedad de discursos o perspectivas teóricas y engloba numerosos métodos y estrategias de recogida de datos. Esta riqueza denota la complejidad y alcance del enfoque cualitativo en el abordaje de la investigación socioeducativa y requiere que se ensayen clasificaciones o categorías que aporten un orden conceptual en el ámbito investigación y permitan la comunicación en la comunidad investigadora. Sin embargo, y 
paradójicamente, cuando se realiza un análisis de las diversas tipologías de modalidades de investigación cualitativa se constata la existencia de una pluralidad de términos usados con diferentes sentidos, que en ocasiones se convierte en una maraña conceptual desconcertante, y que lejos de simplificar esta cuestión, dificulta la tarea de establecer un marco común y homogéneo de clasificación.

Una de las primeras clasificaciones de la investigación cualitativa realizadas fue propuesta por una investigadora del ámbito educativo, Evelyn Jacob (1987), quien identificó cinco tradiciones. La taxonomía inicial de Jacob fue criticada fuertemente por una serie de autores británicos que denunciaron su sesgo antropológico, su reduccionismo al ignorar la realidad de otros estudios desarrollados en contextos no norteamericanos y su fundamentación en los postulados kuhnianos (Atkinson et al., 1988). Posteriormente, Jacob publicaría una revisión de su propuesta añadiendo una sexta tradición y reordenando el listado de las tradiciones dominantes en la investigación educativa (Jacob, 1988). En nuestro país, Cajide (1992) ha realizado una integración de las clasificaciones realizadas por diversos autores y presenta una síntesis de las características de las tradiciones de investigación cualitativa tomando como base la propuesta de Jacob (1988).

Para Pita Fernández, S., \& Pértegas Díaz, S. (2002), el objetivo de cualquier ciencia es adquirir conocimientos y la elección del método adecuado que nos permita conocer la realidad es por tanto fundamental. El problema surge al aceptar como ciertos los conocimientos erróneos o viceversa. Los métodos inductivos y deductivos tienen objetivos diferentes y podrían ser resumidos como desarrollo de la teoría y análisis de la teoría respectivamente. Los métodos inductivos están generalmente asociados con la investigación cualitativa, las características de la investigación cualitativas están orientadas a: Centrada en la fenomenología y comprensión, Observación naturista sin control, es subjetiva, inferencias de sus datos, es exploratoria, inductiva y descriptiva, no generalizable y tiene realidad dinámica, variedad de paradigmas y perspectivas en la investigación cualitativa, Valles, M. S. (2000).

"Un paradigma es una imagen básica del objeto de una ciencia. Sirve para definir lo que debe estudiarse, las preguntas que es necesario responder, cómo deben preguntarse y qué reglas es preciso seguir para interpretar las respuestas obtenidas. El paradigma es la unidad más general de consenso dentro de una ciencia y sirve para diferenciar una comunidad científica (o subcomunidad) de otra. Subsume, define e interrelaciona los ejemplares, las teorías y los métodos e instrumentos disponibles" (1993: 598).

De manera más didáctica y con la atención en la investigación cualitativa, Guba y Lincoln han abordado la definición de los paradigmas en varios de sus escritos (Lincoln y Guba, 1985; Guba, 1990; Guba y Lincoln, 1994). Según estos autores, los paradigmas deben entenderse como sistemas de creencias básicas (principios, supuestos) sobre: a) La naturaleza de la realidad investigada (supuesto ontológico). b) Sobre el modelo de relación entre el investigador y lo investigado (supuesto epistemológico). c) Sobre el modo en que podemos obtener conocimiento de dicha realidad (supuesto metodológico). No se trata, por tanto, de aspectos de método únicamente. El paradigma guía al investigador: además de en la selección de métodos, en aspectos ontológica y epistemológicamente fundamentales (Guba y Lincoln, 1994: 105). La versión de dos paradigmas:

La clasificación más simple de la variedad de paradigmas la proporciona la versión de dos paradigmas contrapuestos. A ellos se refieren las expresiones: Paradigma "prevaleciente", "clásico", "racionalista", "positivista", de un lado. 2) Paradigma "emergente", "alternativo", "naturalista", "constructivista", "interpretativista", de otro.

La versión de cuatro paradigmas: positivismo, postpositivismo, teoría crítica y enfoques afines, constructivismo. A Caballo (1993) entre las versiones dicotómica o tricotómica expuestas y las posibles versiones de cinco o más paradigmas, la clasificación de cuatro tipos de paradigmas da un paso necesario hacia el reconocimiento de la variedad actual de paradigmas en a investigación cualitativa. Los autores que hacen esta propuesta (Guba y Lincoln, 1994) advierten enseguida sobre la categoría clasificatoria más reductora: la que incluye, además de la llamada teoría crítica, al "neomarxismo, feminismo, materialismo y la 
indagación participatoria" (pero sin limitarse a estos). Además, se señala que la teoría crítica podría dividirse a su vez en tres ramas (postestructuralismo, postmodernismo y una combinación de ambos). La agrupación de todos estos enfoques se basa, sobre todo, en dos rasgos comunes que marcarían las diferencias de estos con los paradigmas positivista y postpositivista (frente a los que se consideran alternativos) más que con el constructivismo (paradigma con el que comparten algunos rasgos). Los rasgos comunes a la teoría crítica y enfoques agrupables son:

1) En el componente ontológico, el "realismo histórico". Es decir, la visión de una "realidad virtual configurada por los valores sociales, políticos, culturales, económicos, étnicos y de género" (Guba y Lincoln, 1994: 109). Este realismo histórico se contrapone al "realismo ingenuo" del positivismo y al "realismo crítico" del postpositivismo. Por su parte, el constructivismo queda caracterizado, según estos autores, por un "relativismo" derivado de realidades construidas en contextos concretos. 2) En el componente epistemológico, la creencia de que la investigación (la obtención de conocimiento) está mediada por los valores de un sujeto investigador y un objeto investigado que interactuan (se comunican). Por el contrario, en el positivismo clásico, los resultados de la investigación se consideran ciertos gracias al logro de objetividad, mediante la separación entre el sujeto investigador y el objeto investigado. En cambio, en el postpositivismo los resultados sé consideran probablemente ciertos, y la objetividad se entiende más como un desideratum cuya aproximación requiere replantearse la separación sujeto-objeto. 3) Además de los rasgos ontológicos y epistemológicos, la agrupación de paradigmas alternativos encabezada por la teoría crítica se caracterizaría (según Guba y Lincoln: 1994) por la posición respecto a una serie de cuestiones de orden metodológicopráctico.

Según Pérez Andrés, C. (2000), en los últimos tiempos vienen publicándose en las revistas del ámbito de las ciencias de la salud, tanto nacionales como internacionales, trabajos sobre la metodología cualitativa que incluyen entre sus técnicas las llamadas técnicas de consenso, recientemente, que sólo contempla como técnicas cualitativas la observación participante, la entrevista abierta y los grupos de discusión. Esta situación produce un nivel de confusión que no estaría mal aclarar; es lo que intento al escribir este editorial.

Dentro de las técnicas para el estudio de la realidad social, de la que forman parte los fenómenos relativos a la salud y a la enfermedad, distinguimos entre las llamadas cuantitativas o distributivas y las cualitativas o estructurales. Las primeras describen la realidad a través de una serie de variables con las que se construyen cuestionarios cuyas respuestas sirven para calcular la distribución de la frecuencia de las mismas en las personas que forman la muestra. Cuando se trata de muestras extraídas de la población, estos resultados se pueden inferir al resto de la misma utilizando diferentes cálculos numéricos. Las segundas, que por oposición a éstas, se denominan cualitativas o estructurales, tratan de encontrar la estructura de las relaciones que hacen comprensible el sentido de las representaciones sociales en el lenguaje de los sujetos que forman la sociedad. En concreto, en el ámbito de la salud la metodología cualitativa la explora desde el punto de vista de la concepción cultural y simbólica que la sociedad tiene de la misma.

Pero la principal diferencia entre las técnicas de consenso y las técnicas cualitativas es el tipo de lenguaje con el que ambas trabajan. Los participantes en las técnicas de consenso utilizan el lenguaje científico-técnico, en el cual los códigos utilizados son monosémicos (a un significante le corresponde un solo significado) y denotativos (significados concebidos objetivamente), y la función del lenguaje que prevalece, según el esquema de Jakobson (1963), es la referencial (el mensaje se refiere directamente al objeto, luego es objetivo) y por lo tanto en él no hay nada que interpretar. Al contrario, las técnicas propiamente cualitativas trabajan con el lenguaje social tradicional, cuya función no es sólo referencial sino que incluye la función emotiva (relación entre el mensaje y el emisor), connotativa (relaciones entre el mensaje y el receptor), estética (el objeto de la comunicación es el propio mensaje), fática y metalingüistica (el mensaje tiene por objeto otro mensaje), cuyos códigos son polisémicos (un significante puede remitir a varios significados) y connotativos (conlleva valores subjetivos atribuidos al significante), en los que las representaciones sociales y metáforas constituyen el material que el investigador analiza e interpreta, no para cuantificarlo sino para encontrar el 
sentido oculto que contienen (hermeneútica). Mientras que el saber científico utiliza una codificación por homología (los significantes mantienen entre sí la misma relación que los significados), el saber tradicional se basa en sistemas en los cuales el significante mantiene una relación de analogía (metafórica) con el significado. Así, a diferencia de las técnicas de consenso, la metodología estructural o cualitativa, no sólo no utiliza cuestionarios ni cuantifica las respuestas, sino que analiza e interpreta el lenguaje para encontrar el sentido oculto del fenómeno social que se está investigando, herramienta que no utilizan las técnicas de consenso.

Para, Sampieri, R. H., eat. (1998).La obtención de la información necesaria para la presente investigación es realizada por medio de una INVESTIGACIÓN TECNOLÓGICA: en las ciencias de la ingeniería presenta una serie de características que la vinculan en forma natural con la innovación tecnológica, lo cual indica que las instancias de promoción inicial de los proyectos de investigación y la evaluación de la investigación tecnológica pueden ser utilizadas como un instrumento para fomentar la innovación.- Con innovación tecnológica se designa la incorporación del conocimiento científico y tecnológico, propio o ajeno, con el objeto de crear o modificar un proceso productivo, un artefacto, una máquina, para cumplir un fin valioso para una sociedad.

Es por ello que para la realización del portal web de la parroquia San Nicolás Santuario del Divino Niño Jesús y sus comunidades parroquiales, se ha tomado a bien hacerlo por este método de investigación ya que es el que se considera que satisface las necesidades de nuestra investigación.

\section{MÉtodo de Muestreo}

La muestra se emplea solamente en las ciencias sociales aunque también se puede realizar con animales, dividido en: Estudio de grupo: Para este estudio se emplean muestras grandes de sujetos, seleccionadas por algún método de selección aleatoria, aunque también puede estudiar muestras de sujetos no aleatorios. Se emplea una metodología cuantitativa y los análisis se hace a través de la estadística para generalizar los resultados. En los casos de estudio de

No Probabilístico: éste método no es un tipo de muestreo riguroso y científico, dado que no todos los elementos de la población pueden formar parte de la muestra, se trata de seleccionar a los sujetos siguiendo determinados criterios procurando que la muestra sea representativa. Es decir, los elementos de la muestra son seleccionados por procedimientos al azar o con probabilidades conocidas de selección.

Se aplicará para el Párroco de la iglesia ya que siendo él, el representante oficial de las comunidades parroquiales de la parroquia San Nicolás Santuario del Divino Niño Jesús, es la persona indicada para proporcionar una considerable cantidad de información importante y necesaria para el desarrollo del portal web y también se aplicará para los feligreses que lideran cada ministerio que se desarrolla dentro de las iglesias que conforman la comunidad parroquial.

\section{Tipo de Muestreo}

Para la investigación se utilizará el muestreo de juicio: El investigador toma la muestra seleccionando los elementos que a él le parecen representativos o típicos de la población, por lo que depende del criterio del investigador3, en este caso: se tomarán como muestra a los líderes de cada ministerio dentro de las iglesias de la comunidad parroquial de la Parroquia San Nicolás Santuario del Divino Niño Jesús.

Grupos pequeños, se suelen hacer análisis cualitativos.

Estudio de sujeto único: Se estudia un solo sujeto, es totalmente cualitativo y sus resultados no pueden emplearse para generalizar información

Rodríguez, J. M. (2011) considera que el paradigma de una ciencia se consolida cuando aparece una conceptualización que tenga en cuenta: todos los aspectos del objeto o sujeto de estudio de una ciencia; los 
problemas que deben estudiarse, el método que debe emplearse en la investigación y las formas de explicar, interpretar o comprender, según el caso, los resultados obtenidos por la investigación.

El paradigma surge de un proceso de estudio práctico- teórico; teórico- práctico, ordenado, disciplinado, organizado, permanente y de larga duración en la que se debe comprometer el científico, para comprender/ explicar los conocimientos que ya existen y, sobre la base de estos, edificar nuevos conocimientos que conlleven, como afirma Thomas Kuhn, a nuevas revoluciones científicas. Estas, dada su seriedad y profundidad, se convierten en realizaciones científicas universalmente reconocidas que, durante cierto tiempo, proporcionan modelos de problemas y soluciones a una comunidad científica.

Desde el punto de vista de la comunidad científica un "paradigma” es lo que comparten los miembros de una comunidad científica y, a la inversa una comunidad científica consiste en unas personas que comparten un paradigma (Kuhn, 1977, 271). EL Paradigma explicativo que son:

1. Los fundamentos filosóficos del paradigma explicativo encuentran en el empirismo y en el realismo, especialmente en el realismo científico y crítico, las bases esenciales que lo sustentan. Ambas escuelas afirman la existencia de cosas reales, independientes de la conciencia. Sin embargo, se reconoce, en estas escuelas, que el objeto percibido no es una mera reproducción de la realidad, pues en el proceso de conocimiento se combinan la percepción y el pensamiento.

2. Todo conocimiento empírico se relaciona de una forma u otra con alguna observación directa, pero las teorías y las hipótesis permiten observaciones indirectas que proporcionan conocimientos más amplios y profundos.

3. Sólo es posible conocer entes materiales y objetos conceptuales, es decir, conceptos, proposiciones y sistemas relacionados de proposiciones. El conocimiento puede referirse a entes individuales o a relaciones que se dan entre los fenómenos.

Guías de observación, para las cuales es importante tener en cuenta los siguientes pasos: precisar lo que se va a observar; categorizar lo que se desea observar después de un primer acercamiento a la comunidad; ubicar los aspectos que se van a observar en la dimensión que se desea; el problema; y el objetivo general. La guía debe estar acorde con el problema planteado en la investigación y el objetivo general propuesto.

- La encuesta. A través de este método se obtiene información de los grupos que se estudian. Ellos mismos proporcionan la información sobre sus actitudes, opiniones, sugerencias, etc. Existen dos maneras de obtener información con este método: la entrevista y el cuestionario.

- La entrevista. La entrevista es simplemente la comunicación interpersonal establecida entre el investigador y el sujeto de estudio con un determinado propósito. Es una relación que tiene por objeto obtener respuestas verbales a los interrogantes planteados sobre el problema propuesto. Está orientada a recolectar datos que tienen que ver con las percepciones, las actitudes, las opiniones, las experiencias ya vividas, los conocimientos, así como también a los proyectos de futuro. La entrevista es una técnica personal que permite la recolección de la información en profundidad donde el informante expresa o comparte oralmente y por medio de una relación interpersonal con el investigador su saber (opiniones, creencias, sentimientos, puntos de vista y actitudes) respecto de un tema o hecho.

\section{CONCLUSIONES}

Podemos concluir que para la medición del impacto socio económico del Bono productivo en Nicaragua, es conveniente utilizar una metodología investigación participativa que nos permita conocer de la experiencia compartida de los beneficiarios del Bono y para ello es conveniente utilizar un método cuali- participativo. La investigación va más allá de los límites de un individuo investigador para ubicarse en redes de problemas, temas e intereses que muchas veces abarcan largos períodos históricos y varias generaciones de estudiosos, constituyendo así una visión transindividual de cualquier proceso de investigación. Cuando un investigador elige un problema de estudio dentro de una temática determinada, lo que hace es inscribirse dentro de una 
red temática y problemática en la que también trabajan y han estado trabajando otros investigadores. Esta red suelen tener en el tiempo toda una trayectoria de desarrollo y que, a su vez, mantiene sucesivas y complejas conexiones con otras redes y su Programa de Investigación o Líneas de trabajo, que remite a secuencias de desarrollo en torno a un problema global y que aglutina a Grupos Académicos. Galileo y Einstein ilustraron perfectamente bien este concepto cuando declararon que el éxito de sus trabajos personales se debía al hecho de haber caminado "sobre hombros de gigantes", refiriéndose precisamente a que retomaron planteamientos previamente formulados y se basaron en intentos y soluciones anteriores a ellos, sin tener que comenzar desde cero (Padrón, 1998). No obstante, escogencia correcta del tipo de investigación juega un papel crucial para el éxito de toda investigación, así como también, el método, y el paradigma que se empleara en su conjunto conforman la columna vertebral de todo proceso investigativo.

\section{LITERATURA CITADA}

Atkinson, H. C., \& Begg, E. J. (1988). The binding of drugs to major human milk whey proteins. British journal of clinical pharmacology, 26(1), 107-109.

Cajide Val, J. (1992). El desarrollo intelectual en matemáticas: perspectivas desde una experimentación. Educadores: Revista de la Federación Española de Religiosos de Enseñanza, 34(161), 67-73.

CABALLO, V. E. (1993). Manual de evaluación y entrenamiento de las habilidades sociales.

Guba, E. G., \& Lincoln, Y. S. (1994). Competing paradigms in qualitative research. Handbook of qualitative research, 2(163-194), 105.

Hernández, R., Fernández, C. B., \& Baptista, L. P.(2003). Metodología de la Investigación, (Vol. 3).

Hernández Sampieri, R., Fernández Collado, C., \& Baptista Lucio, P. (1991). Metodología de la Investigación. 2 da. Edición. Editorial Mc Graw Hill.

Hernández, R., \& Fernández, C. B. (2003) Metodología de la investigación. Editorial Mc Graw Hill. México.

Hernández, R., Fernández, C., \& Baptista, P. (2006). Metodología de la investigación.

Guba, E. G. (1990). The paradigm dialog. In Alternative paradigms conference, mar, 1989, indiana u, school of education, san francisco, ca, us. Sage Publications, Inc.

Jakobson, R. (1963). Essais de linguistique générale.

Kuhn, T. S. (1977). The Essential Tension. Selected Studies in Scientific Tradition and Change. Chicago: University of Chicago Press.

Lincoln, Y., \& Guba, E. (1985). Naturalistic Inquiry Vol. 75 Sage. Beverly Hills, CA.

Méndez, I., Namihira, D., Moreno, L., \& Sosa, C. (2001). El protocolo de investigación. México DF: Trillas.

Padrón, J. (1998). La estructura de los procesos de investigación. Revista educación y ciencias humanas, 9(17), 33-45.

Paz, M. (2003). Investigación cualitativa en educación. Fundamentos y tradiciones. Editorial Mcgraw Hill. México DF.

Pita Fernández, S., \& Pértegas Díaz, S. (2002). Investigación cuantitativa y cualitativa. Cad aten primaria, 9(76-8).

Pérez Andrés, C. (2000). ¿ Deben estar las técnicas de consenso incluidas entre las técnicas de investigación cualitativa?. Revista Española de Salud Pública, 74(4), 00-00.

Rodríguez, J. M. (2011). Métodos de investigación cualitativa. Revista Silogismo, 4(8), 11-11.

Sampieri, R. H., Collado, C. F., Lucio, P. B., \& Pérez, M. D. L. (1998). Metodología de la investigación (Vol. 1). McGraw-Hill.

Valles, M. S. (2000). Técnicas cualitativas de investigación social. Madrid: Sintesis Editorial. 